American J. of Engineering and Applied Sciences 1 (3): 219-222, 2008

ISSN 1941-7020

(C) 2008 Science Publications

\title{
A Novel Integrated SET Based Inverter for Nano Power Electronic Applications
}

\author{
Negin Moezi, Daryoosh Dideban and Abbas Ketabi \\ Department of Electrical Engineering, University of Kashan, Kashan, Iran
}

\begin{abstract}
In this research an emerging field of power electronics in nanotechnology is survived. This application-based technology today is called Nano Power Electronics. It utilizes nano electronic transistors in switching applications in the range of nano power for signal shaping purposes. In recent years Single Electron Transistors are highly interested in nano electronic applications. They have got inherently fast tunneling rate, which makes them highly suitable for high-speed operation. Based on this fact, a novel nano DC/AC converter nano inverter is proposed with these transistors. Simulation of this integrated nano inverter with SPICE shows it has excellent output waveforms and it will be a good candidate for nano power electronic applications.
\end{abstract}

Key words: Nano power electronics, nano inverter, nano transistor, single electron tunneling, spice macro modeling

\section{INTRODUCTION}

Nano power electronics is a new emerging field of technology in the twenty first century. The aims of nano power electronics are power shaping (from DC to AC) for nano electro mechanic loads. Applications of nano power electronics are in controlling the speed of nano motors, automotive micro and nano systems, medical technology, nano robotics and future space systems ${ }^{[1-3]}$. These applications are interested because of the attention, which is focused to reduction of size, cost and power loss.

Advanced semiconductor technology continues to drive the scaling of electronic device dimensions into the ultra thin and nano dimensional regime. The main focus of nano scale power electronics is to use this nano sized semiconductors in power electronic circuits. Thus nano power electronics is a multidisciplinary field which it's study has some prerequisites such as quantum science for advanced nanometer regime semiconductors, power electronic systems (switching converters), control theory and VLSI integration technology. Figure 1 shows these sources of nano power electronics study and some of it's most important applications. The area of nano scale power electronic devices and elements largely includes all types of newly being formulated tunneling devices and elements. The dimensions of such devices are in the range of few nanometers and they are the most promising candidates for the use in all nano power electronic application areas. Such devices are being widely utilized as single

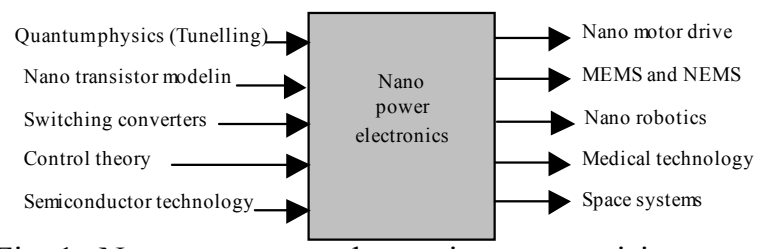

Fig. 1: Nano power electronic prerequisites and applications

electron devices for the production of Ultra-LargeScale-Integration (ULSI) circuits and devices.

\section{MATERIALS AND METHODS}

Using set as a nano switch: Current progress in electronics industry is pushing Metal Oxide Semiconductor Field Effect Transistor (MOSFET) dimensions towards the $10-\mathrm{nm}$ gate length limit, which is expected to be the basic physical limit of conventional MOSFETs ${ }^{[4]}$. When semiconductor structures are scaled down to the nanometer region and beyond, quantum mechanics effects increases and finally determine the behavior. While for CMOS this causes faults to occurand therefore is a limit to the scalability, for SET this means scalability even on atomic scale set, because SET is based on quantum mechanics effects.

To ensure further feature-size reduction, one possible solution is to develop Single-Electron Tunneling (SET) devices. SET devices and circuits have been developed rapidly in both theory and applications during the past two decades. 


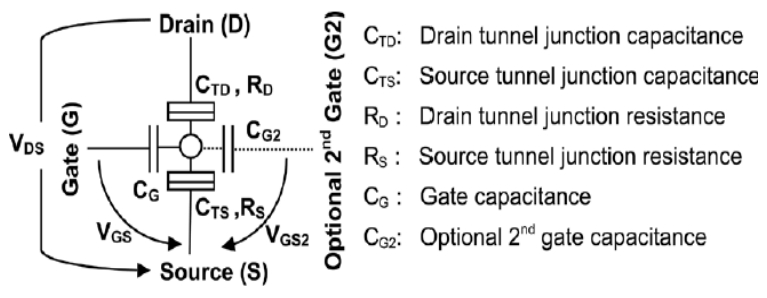

Fig. 2: Schematic of a basic single electron transistor and its different parameters

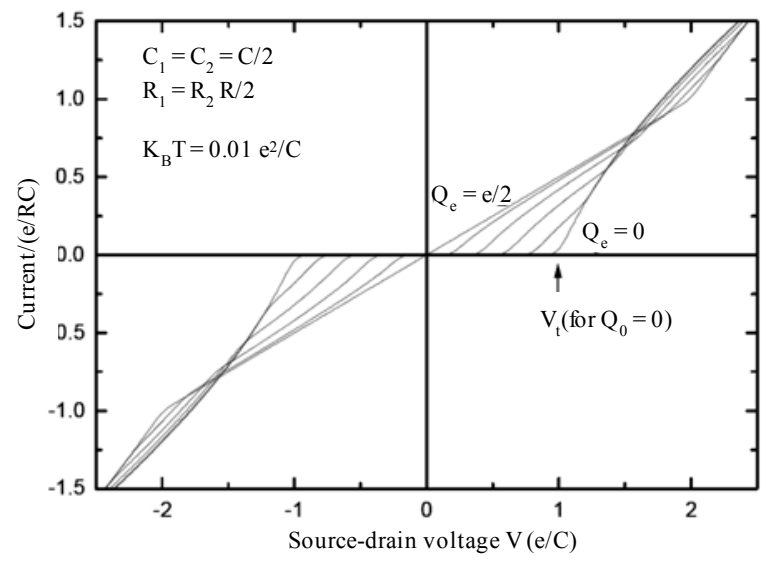

Fig. 3: Current-voltage characteristic of basic SET device under different gate voltages

The fundamental principle of SET device action is the coulomb blockade phenomena, which result in on or off states of this transistor ${ }^{[5-7]}$. Some recent applications of SET are switching based such as analog to digital and digital to analog converters ${ }^{[8]}$ and nanometer digital gate design ${ }^{[9]}$.

The basic schematic of a SET device, where a conductive island is sandwiched between two tunnel junctions is shown in Fig. 2. A proper operation of a SET device requires: (1) the tunnel junction resistance $\left(R_{D}, R_{S}\right)$ to be greater than the quantum resistance to confine the electrons in the islandand (2) the charging energy of the island capacitance to be larger than the available thermal energy to avoid electron tunneling due to the thermionic emission ${ }^{[5]}$.

The current-voltage characteristic of this device is shown in Fig. 3 for different gate voltages ${ }^{[10]}$. Note that in off state, device is in coulomb blockade and no current flows through it but with an increase in drainsource voltage after reaching a critical voltage, device can conduct and will be in on state. The effect of gate voltage is lowering the critical drain-source voltage needed to start conducting the current. The relationship between $\mathrm{Q}_{0}$ (external charge of island) and gate voltage

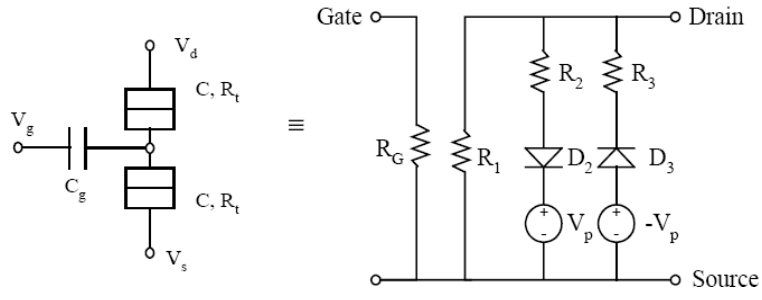

Fig. 4: Macro-modeling of an SET(equivalent circuit of an SET)

is linear with a parameter constant of $\mathrm{C}_{\mathrm{G}}$ (gate capacitance). Optional second gate showed on Fig. 1 is for trimming and adjusting the background (internal) charge of island.

\section{RESULTS AND DISCUSSION}

SET macromodeling for nano power electronic applications: A few circuit models are proposed in the literatures. One of them that best fits with switching applications is presented in $^{[11]}$. Figure 4 shows the proposed SET equivalent circuit which can be converted to SPICE code. Symmetric features of the drain-source current-voltage (Ids-Vds) characteristics are incorporated with two branches consisting of the combination of resistors, diodesand voltage sources. They are denoted by $\mathrm{R} 2 / \mathrm{D} 2 / \mathrm{V} 2$ and $\mathrm{R} 3 / \mathrm{D} 3 / \mathrm{V} 3$, respectively. The directions of D2 and V2 are opposite to those of D3 and V3 to have the current flow in both positive and negative drain-source bias. The charging energy, periodically changing as a function of the gate bias, is included in R1, R2 and R3 where the cosine of the gate bias is used. They are expressed as follows:

$$
\begin{gathered}
\mathrm{R}_{1}\left(\mathrm{~V}_{\mathrm{g}}\right)=\mathrm{CR} 1+\mathrm{CR} 2 \cdot \operatorname{Cos}\left(\mathrm{CF} 1 . \mathrm{V}_{\mathrm{g}}\right) \\
\mathrm{R}_{2}\left(\mathrm{~V}_{\mathrm{g}}\right)=\mathrm{R}_{3}\left(\mathrm{~V}_{\mathrm{g}}\right)=\frac{\mathrm{CVp}}{\mathrm{CI} 2-2 \mathrm{CVp} / \mathrm{R}_{1}\left(\mathrm{~V}_{\mathrm{g}}\right)}
\end{gathered}
$$

Note that all of resistors in the macro model are highly nonlinear so we expect having challenges in the simulation time and speed.

The parameters, CF1, CVp, CI2, CR1and CR2 are used to fit the current-voltage characteristics at various gate biases.

A novel set based half bridge nano inverter: Half Bridge DC/AC converter which is named inverter is simplest switching converter which is survived in all texts $^{[12]}$. 


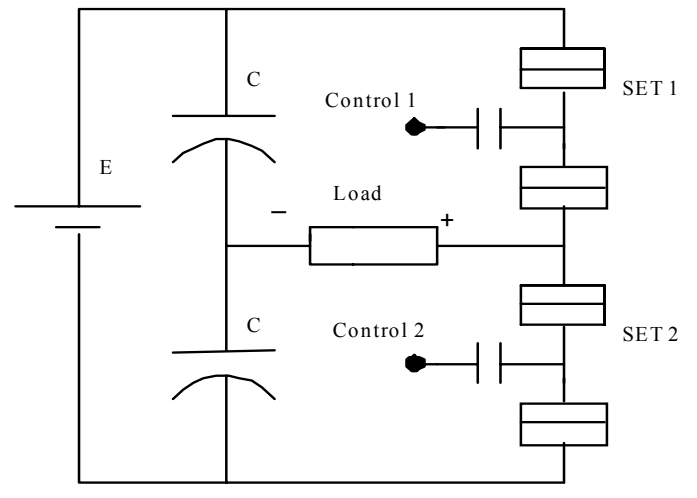

Fig. 5: A half bridge Nano Inverter utilizing SETs

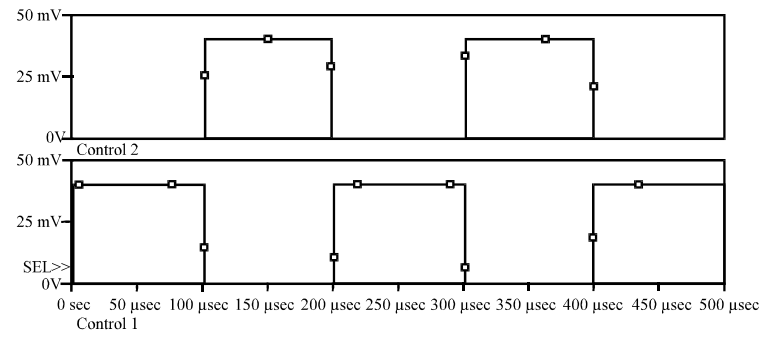

Fig. 6: Control signals of proposed nano inverter

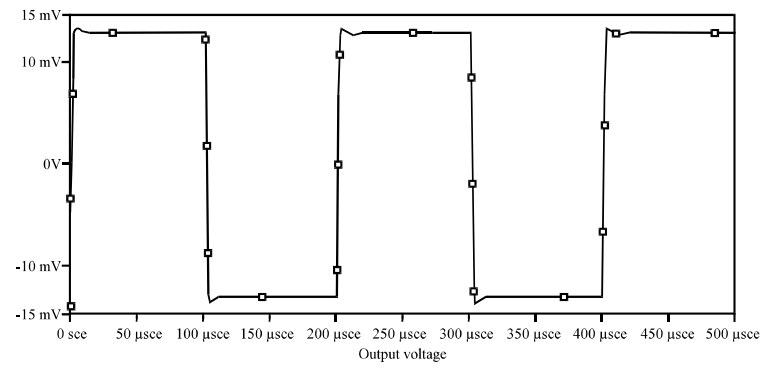

Fig. 7: Output (Load) voltage of proposed Nano Inverter for $\mathrm{E}=30 \mathrm{mv}$ and resistive load

Figure 5 shows a novel half bridge inverter which is suitable for nano power applications. In this circuit SETs act as switches. Control signals of SETs are pulses driving nano switch in on or off regions as shown in Fig. 6. These pulses have a frequency of $5 \mathrm{KHz}$ and a height of $50 \mathrm{mv}$ which is sufficien to switch the SET's. SPICE simulation results of Fig. 5 for the output (load) voltage is shown in Fig. 7.

There is no limitation in theory for switching speed of SETs compared to nano MOSFETs but in practice, load and interconnection parasites of transistors cause a limit on the switching frequency of nano inverter.

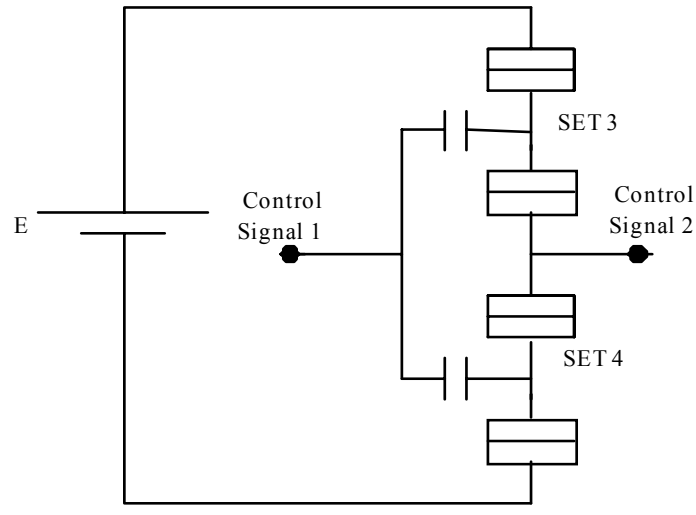

Fig. 8: SET based digital Inverter for derivation of control signal 2

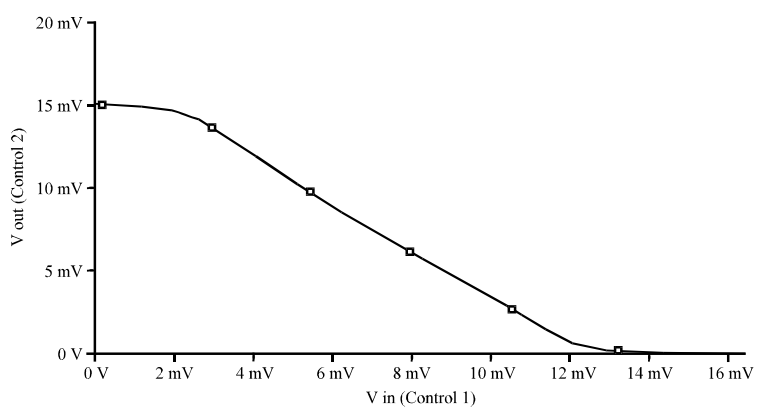

Fig. 9: Input-output characteristics of SET based digital inverter (Fig. 8)

The output waveform is with good agreement with known conventional switch based half bridge inverter. Control signal 2 can be derived from control signal 1 utilizing a NOT gate. This digital gate can be implemented with two series SETs as shown in Fig. 8 which is biased by the same DC input voltage. The final goal is to have an integrated nano inverter. The inputoutput characteristic of this circuit is simulated and shown on Fig. 9 which is in good agreement with conventional digital inverters.

\section{CONCLUSION}

Nano scale power electronics will immensely advance all of the future applications. This study addressed one kind of novel nano power electronic converter, which consists of SET transistors. We used SPICE macro-modeling for SETs as switches in a nano inverter. We simulated proposed half bridge nano inverter and the simulation results demonstrated that the SETs can be used as good nano switches in all of nano power electronic applications. These transistors had no theoretical limitations in switching speed in contrast to 
nano MOSFETs although circuit and interconnection parasitics set an upper limitation for them. Also they can be used for the integration of power circuit and control circuit in nano power electronic applications.

\section{REFRENCES}

1. A.Chakraborty, A.Emadi "Quantum sizing of power electronics: A trend towards miniaturization of power electronic systems and equipments" Material Research Society Symposium Proceedings, Vol.872 , 2005, Materials Research Society, pages : J18.28.1-J18.28.6.

2. A.Chakraborty, A.Emadi "Application and research on nano power electronics: An adventure beyond quantum electronics" Proceedings of SPIE, Vol 5824, 2005, doi : 10.1117/12.604791, pages : 260-268.

3. S.Irudayaraj, A.Chakraborty, A.Emadi "Micro and nano scale electric machines and applications of power electronics" Power Electronics Specialists Conference,2005,IEEE,Vol36,Pages:1191-1197 doi : 10.1109/PESC.2005.1581780

4. C.H.Hu,S.D.Cotofana,J.F.Jiang "Digital to analogue converter based on single electron tunneling transistor" IEE Proceedings on Circuits, Devices and Systems, Vol 151, No.5, October 2004,Pages:438-442, doi:10.1049/ip-cds:20040992

5. S.Mahapatra, V.Vaish et al."Analytical modeling of single electron transistor for hybrid CMOS-SET analog IC design" IEEE Trans. Elect. Devices, Vol 51, No.11, November 2004, Pages:1772-1782, doi: 10.1109/TED.2004.837369
6. J.Hoekstra "On circuit theories for single-electron tunneling devices" IEEE Transactions on Circuits and Systems, Volume 54, Issue 11, Nov. 2007, Pages:2353-2359, doi: 10.1109/TCSI.2007.907797

7. J.Hoekstra "Towards a circuit theory for metallic single-electron tunneling devices" International Journal of Circuit Theory and Applications, Published online 20 April 2007 in Wiley InterScience (www.interscience.wiley.com), Pages: 213-238, doi: 10.1002/cta.412

8. X.Ou, N.J.Wu, "Analog-digital and digital-analog converters using single electron and MOS transistors"IEEE Transactions on Nanotechnology, VOL.4, NO.6, NOVEMBER 2005, doi: 10.1109/TNANO.2005.858600

9. A.Venkataratnam,A.K.Goel"Design and simulation of logic circuits with hybrid architectures of single electron transistors and conventional devices" first International Conference on Nano-Networks and workshops, September 2006, Pages:1-5, doi: 10.1109/NANONET.2006.346218

10. K.K.Likharev "Single-electron devices and their applications" Proc. IEEE, Volume 87, Issue 4, April 1999, Pages:606-632, doi: 10.1109/5.752518

11. Y.S.Yu,H.S.Leeand,S.W.Hwang "SPICE macro modeling for the compact simulation of single electron circuits" Journal of Korean Physics Society, Vol 33, Pages:269-279, 1998

12. A.Ketabi,D.Dideban,"PowerElectronics: Elements, Circuits and Applications" Morsal Publications (Published in Persian), Edition 1, May 2008, ISBN: 978-964-972-042-5 